\title{
The asymptotic behavior of a stochastic SIS epidemic model with vaccination
}

\author{
Yanan Zhao ${ }^{1,2}$, Qiumei Zhang ${ }^{1,2}$ and Daqing Jiang ${ }^{1,3^{*}}$
}

\section{"Correspondence:}

daqingjiang2010@hotmail.com

'School of Mathematics and

Statistics, Northeast Normal

University, Changchun, Jilin 130024,

P. R. China

${ }^{3}$ College of Science, China

University of Petroleum (East China),

Qingdao, 266580, China

Full list of author information is

available at the end of the article

\begin{abstract}
In this paper, we discuss a stochastic SIS epidemic model with vaccination. We investigate the asymptotic behavior according to the perturbation and the reproduction number $R_{0}$. When the perturbation is large, the number of infected decays exponentially to zero and the solution converges to the disease-free equilibrium regardless of the magnitude of $R_{0}$. Moreover, we get the same exponential stability and the convergence if $R_{0}<1$. When the perturbation and the disease-related death rate are small, we derive that the disease will persist, which is measured through the difference between the solution and the endemic equilibrium of the deterministic model on average in time if $R_{0}>1$. Furthermore, we prove that the system is persistent in the mean. Finally, the results are illustrated by computer simulations.
\end{abstract}

MSC: $34 \mathrm{~F} 05 ; 37 \mathrm{H} 10 ; 60 \mathrm{H} 10 ; 92 \mathrm{D} 25 ; 92 \mathrm{D} 30$

Keywords: stochastic SIS epidemic model; vaccination; exponential stability; persistent in mean; Lyapunov function

\section{Springer}

\section{Introduction}

Epidemiology is the study of the spread of diseases with the objective to trace factors that are responsible for or contribute to their occurrence. Significant progress has been made in the theory and application of epidemiology modeling by mathematical research. In a simple epidemic model, there is generally a threshold, $R_{0}$. If $R_{0} \leq 1$, the disease-free equilibrium is a unique equilibrium in this type of epidemic model and it is globally asymptotically stable; if $R_{0}>1$, this type of model has also a unique endemic equilibrium, which is globally asymptotically stable. Therefore, the threshold $R_{0}$ determines the extinction and persistence of the epidemic.

Controlling infectious diseases has been an increasingly complex issue in recent years. Vaccination is an important strategy for the elimination of infectious diseases [1-3]. The vaccination enables the vaccinated to acquire a permanent or temporary immunity. When the immunity is temporary, the immunity can be lost after a period of time. It is used in many references [4-7] where one assumes the process of losing immunity is in the exponential form.

(c) 2015 Zhao et al. This article is distributed under the terms of the Creative Commons Attribution 4.0 International License (http://creativecommons.org/licenses/by/4.0/), which permits unrestricted use, distribution, and reproduction in any medium, provided you give appropriate credit to the original author(s) and the source, provide a link to the Creative Commons license, and indicate if changes were made. 
Li and Ma [7] discussed a SIS model with vaccination. The system has following form:

$$
\left\{\begin{array}{l}
\dot{S}(t)=(1-q) A-\beta S(t) I(t)-(\mu+p) S(t)+\gamma I(t)+\varepsilon V(t) \\
\dot{I}(t)=\beta S(t) I(t)-(\mu+\gamma+a) I(t) \\
\dot{V}(t)=q A+p S(t)-(\mu+\varepsilon) V(t) .
\end{array}\right.
$$

Here $S(t)$ denotes the number of susceptible individuals at time $t, I(t)$ denotes the number of infected individuals at time $t$ and $V(t)$ denotes the number of members who are immune to an infection as the result of vaccination at time $t$. The parameters in the model are summarized in the following list:

$A$ : the constant input of new members into the population per unit time;

$q$ : the fraction of vaccinated new-borns;

$\beta$ : transmission coefficient between compartments $S$ and $I$;

$\mu$ : the natural death rate of the $S, I, V$ compartments;

$p$ : the proportionality coefficient of vaccinated cases for the susceptible;

$\gamma$ : the recovery rate of infectious individuals;

$\varepsilon$ : the rate of losing their immunity for vaccinated individuals;

$\alpha$ : the disease-caused death rate of infectious individuals.

All parameter values are assumed to be nonnegative and $\mu, A>0$.

According to the theory in $\mathrm{Li}$ and $\mathrm{Ma}$ [7], system (1.1) always has the disease-free equilibrium $P_{0}=\left(S_{0}, I_{0}, V_{0}\right)=(A(\mu(1-q)+\varepsilon) / \mu(\mu+\varepsilon+p), 0, A(\mu q+p) / \mu(\mu+\varepsilon+p))$. If $R_{0} \leq 1$, then $P_{0}$ is the unique equilibrium of (1.1) and it is globally stable in the invariant set $\Gamma$, where $\Gamma=\{(S, I, V): S>0, I \geq 0, V \geq 0, S+I+V \leq A / \mu\}$. If $R_{0}>1$, then $P_{0}$ is unstable and there is an endemic equilibrium $P^{*}=\left(S^{*}, I^{*}, V^{*}\right)=((\mu+\gamma+\alpha) / \beta, \mu(\mu+\gamma+\alpha)(\mu+$ $\left.\varepsilon+p)\left(R_{0}-1\right) / \beta(\mu+\alpha)(\mu+\varepsilon),(q A+p(\mu+\gamma+\alpha) / \beta) /(\mu+\varepsilon)\right)$ of system (1.1), which is globally asymptotically stable under a sufficient condition in $\Gamma$. The reproduction number of system (1.1)

$$
R_{0}=\frac{A \beta(\mu(1-q)+\varepsilon)}{\mu(\mu+\gamma+\alpha)(\mu+\varepsilon+p)}
$$

However, the deterministic approach has some limitations in the mathematical modeling of the transmission of an infectious disease and it is quite difficult to predict the future dynamics of a system accurately. This happens due to the fact that deterministic models do not incorporate the effect of a fluctuating environment. Stochastic differential equation models play a significant role in various branches of applied sciences including infectious dynamics, as they provide some additional degree of realism compared to their deterministic counterpart. In reality, parameters involved in the modeling approach of ecological systems are not absolute constants, and they always fluctuate around some average value due to continuous fluctuations in the environment. As a result, parameters in the model never attain a fixed value with the advancement of time and rather exhibit a continuous oscillation around some average values. Many authors have introduced parameters of random perturbation into epidemic models and have studied their dynamics. 
Parameter perturbations on the transmission rate $\beta$ are considered in [8-12]. Tornatore et al. in [9] studied the following stochastic SIR model with no delay:

$$
\left\{\begin{array}{l}
d S(t)=[\mu-\mu S(t)-\beta S(t) I(t)] d t-\sigma S(t) I(t) d B(t), \\
d I(t)=[\beta S(t) I(t)-(\mu+\gamma) I(t)] d t+\sigma S(t) I(t) d B(t), \\
d R(t)=[\gamma I(t)-\mu R(t)] d t .
\end{array}\right.
$$

They proved that $0<\beta<\min \left\{\gamma+\mu-\sigma^{2} / 2,2 \mu\right\}$ was a sufficient condition for the asymptotic stability of the disease-free equilibrium. Using only computer simulations they showed that if $\min \left\{\gamma+\mu-\sigma^{2} / 2,2 \mu\right\}<\beta<\gamma+\mu+\sigma^{2} / 2$, the disease-free equilibrium was stable and the disease does not occur. If $(\gamma+\mu)\left(R_{0}-1\right)>\sigma^{2} / 2$, the disease-free equilibrium was unstable. The same discussion was extended to a SIRS model by Liu [10]. By using Lyapunov functions, in [11] was shown the asymptotic behavior of a multigroup SIR epidemic model with fluctuations around parameter $\beta$. When $R_{0} \leq 1$, one deduced the globally asymptotic stability of the disease-free equilibrium $P_{0}$, which meant the disease would die out. When $R_{0}>1$ and the perturbation was small, by measuring through the difference between the solution and the endemic equilibrium of the deterministic model in time average, they derived that the disease would persist. However, the authors did not consider the case when the perturbation would be large. Besides, Gray et al. in [12] discussed the following stochastic SIS model:

$$
\left\{\begin{array}{l}
d S(t)=[\mu N-\beta S(t) I(t)+\gamma I(t)-\mu S(t)] d t-\sigma S(t) I(t) d B(t), \\
d I(t)=[\beta S(t) I(t)-(\mu+\gamma) I(t)] d t+\sigma S(t) I(t) d B(t) .
\end{array}\right.
$$

They proved that this model had a unique global positive solution and established conditions for extinction and persistence of $I(t)$ according to the perturbation and the reproductive number $R_{0}$. In the case of persistence they showed the existence of a stationary distribution and derived expressions for its mean and variance. Besides the parameters with the stochastic perturbation, there are other types of stochastic perturbations; see [13-19].

Jing $\mathrm{Fu}$ et al. introduced stochasticity into a multigroup SIS model in [25]. They presented the sufficient condition for the exponential extinction of the disease and proved that the noises significantly raise the threshold of a deterministic system. In the case of persistence, they proved that there exists an invariant distribution which is ergodic.

In [26], Golmankhaneh et al. applied the homotopy analysis method (HAM) successfully for solving second-order random differential equations, homogeneous or inhomogeneous. Expectation and variance of the approximate solutions were computed. Several numerical examples were presented to show the ability and efficiency of this method. Jafarian et al. in [27] solved linear second kind Fredholm and Volterra integral equations systems by applying the Bernstein polynomials expansion method. Illustrative examples were provided to demonstrate the preciseness and effectiveness of the proposed technique.

Another approach to include stochastic perturbations in a biological model was considered by Imhof and Walcher in [23]. Applying this method, Zhao and Jiang [24] discussed 
the stochastic system

$$
\left\{\begin{array}{l}
\dot{S}(t)=[(1-q) A-\beta S I-(\mu+p) S+\gamma I+\varepsilon V] d t+\sigma_{1} S d B_{1}(t) \\
\dot{I}(t)=[\beta S-(\mu+\gamma+\alpha)] I d t+\sigma_{2} I d B_{2}(t) \\
\dot{V}(t)=[q A+p S-(\mu+\varepsilon) V] d t+\sigma_{3} V d B_{3}(t)
\end{array}\right.
$$

where $B_{i}(t)$ are independent Brownian motions, and $\sigma_{i}(i=1,2,3)$ are their intensities. When the perturbations and the disease-related death rate $\alpha$ are small, they showed that there is a stationary distribution and it is ergodic as $R_{0}>1$ and the asymptotic behavior of the solution around the disease-free equilibrium $P_{0}$ as $R_{0} \leq 1$. Here $R_{0}$ is the reproductive number of the deterministic model.

In this paper, taking into account the effect of a randomly fluctuating environment, we assume that fluctuations in the environment will manifest themselves mainly as fluctuations in the parameter $\beta$, as in [12],

$$
\beta \rightarrow \beta+\sigma \dot{B}(t)
$$

where $B(t)$ is standard Brownian motions with $B(0)=0$, and with the intensity of the white noise $\sigma^{2}>0$. The stochastic version corresponding to the deterministic model (1.1) takes the following form:

$$
\left\{\begin{array}{l}
d S(t)=((1-q) A-\beta S(t) I(t)-(\mu+p) S(t)+\gamma I(t)+\varepsilon V(t)) d t-\sigma S(t) I(t) d B(t) \\
d I(t)=(\beta S(t) I(t)-(\mu+\gamma+\alpha) I(t)) d t+\sigma S(t) I(t) d B(t), \\
d V(t)=(q A+p S(t)-(\mu+\varepsilon) V(t)) d t
\end{array}\right.
$$

The main objective of this paper is to study not only when the disease will die out and but also when it will persist according to the perturbation and the threshold $R_{0}$.

This paper is organized as follows. In Section 2, we show there is a unique positive solution of system (1.3) for any positive initial value. In Section 3, we show that the positive solution of system (1.3) converges to $P_{0}$ exponentially as the perturbation is large. In Section 4 , we get the same exponential stability and the convergence when $R_{0}<1$. On the other hand we investigate the asymptotic behavior of the solution of the system (1.3) according to $R_{0}>1$ although the solution of system (1.3) does not converge to $P^{*}$. When the perturbation is not large, we consider the disease to persist. Moreover, we show system (1.3) is persistent in the mean. The key to our analysis is choosing appropriate Lyapunov functions. In Section 5, we make simulations to conform our analytical results. In Section 6, we give a short conclusion. Finally, in order to be self-contained, we have an Appendix containing a lemma used in the previous sections.

\section{Existence and uniqueness of positive solution}

To investigate the dynamical behavior, the first concern is whether the solution has a global existence. Moreover, for a population dynamics model, whether the value is positive is also considered. Hence in this section we first show that the solution of system (1.3) is global and positive. As we know, in order to get a stochastic differential equation which has a unique global (i.e. no explosion in finite time) solution for any given initial value, the 
coefficients of the equation are generally required to satisfy the linear growth condition and local Lipschitz condition (cf. [20]). However, the coefficients of system (1.3) do not satisfy the linear growth condition, though they are locally Lipschitz continuous, so the solution of system (1.3) may explode at a finite time. In this section, using the Lyapunov analysis method (mentioned in [11-13]), we show the solution of system (1.3) is positive and global.

Theorem 2.1 There is a unique solution $(S(t), I(t), V(t))$ of system (1.3) on $t \geq 0$ for any initial value $(S(0), I(0), V(0)) \in \mathbb{R}_{+}^{3}$, and the solution will remain in $\mathbb{R}_{+}^{3}$ with probability 1 , namely, $(S(t), I(t), V(t)) \in \mathbb{R}_{+}^{3}$ for all $t \geq 0$ almost surely.

Proof Since the coefficients of the equation are locally Lipschitz continuous for any given initial value $(S(0), I(0), V(0)) \in \mathbb{R}_{+}^{3}$, there is a unique local solution $(S(t), I(t), V(t))$ on $t \in\left[0, \tau_{e}\right)$, where $\tau_{e}$ is the explosion time (in the Appendix). To show that this solution is global, we need to show that $\tau_{e}=\infty$ a.s. Let $k_{0} \geq 0$ be sufficiently large so that $S(0), I(0)$ and $V(0)$ all lie within the interval $\left[1 / k_{0}, k_{0}\right]$. For each integer $k \geq k_{0}$, define the stopping time

$$
\tau_{k}=\inf \left\{t \in\left[0, \tau_{e}\right): \min \{S(t), I(t), V(t)\} \leq \frac{1}{k} \text { or } \max \{S(t), I(t), V(t)\} \geq k\right\}
$$

where, as throughout this paper, we set $\inf \emptyset=\infty$ (as usual $\emptyset$ denotes the empty set). According to the definition, $\tau_{k}$ is increasing as $k \rightarrow \infty$. Set $\tau_{\infty}=\lim _{k \rightarrow \infty} \tau_{k}$, whence $\tau_{\infty} \leq \tau_{e}$ a.s. If we can show that $\tau_{\infty}=\infty$ a.s., then $\tau_{e}=\infty$ and $(S(t), I(t), V(t)) \in \mathbb{R}_{+}^{3}$ a.s. for all $t \geq 0$. In other words, to complete the proof all we need to show is that $\tau_{\infty}=\infty$ a.s. If this statement is false, then there exist a pair of constants $T>0$ and $\varepsilon \in(0,1)$ such that

$$
P\left\{\tau_{\infty} \leq T\right\}>\varepsilon
$$

Hence there is an integer $k_{1} \geq k_{0}$ such that

$$
P\left\{\tau_{k} \leq T\right\} \geq \varepsilon \quad \text { for all } k \geq k_{1} .
$$

For $t \leq \tau_{k}$, we can see, for each $k$,

$$
d(S+I+V)=[A-\mu(S+I+V)-\alpha I] d t \leq[A-\mu(S+I+V)] d t
$$

and so

$$
S(t)+I(t)+V(t) \leq\left\{\begin{array}{ll}
\frac{A}{\mu}, & \text { if } S(0)+I(0)+V(0) \leq \frac{A}{\mu} \\
S(0)+I(0)+V(0), & \text { if } S(0)+I(0)+V(0)>\frac{A}{\mu}
\end{array}:=M .\right.
$$

Define a $C^{2}$-function $W: \mathbb{R}_{+}^{3} \rightarrow \overline{\mathbb{R}}_{+}$by

$$
W(S, I, V)=(S-1-\log S)+(I-1-\log I)+(V-1-\log V) .
$$


Let $k \geq k_{0}$ and $T>0$ be arbitrary. Applying the Itô formula, we obtain

$$
\begin{aligned}
d W(S, I, V) & =\left(1-\frac{1}{S}\right) d S+\frac{1}{2 S^{2}}(d S)^{2}+\left(1-\frac{1}{I}\right) d I+\frac{1}{2 I^{2}}(d I)^{2}+\left(1-\frac{1}{V}\right) d V \\
& =L W d t+\sigma(I-S) d B(t),
\end{aligned}
$$

where $L W: \mathbb{R}_{+}^{3} \rightarrow \mathbb{R}_{+}$is defined by

$$
\begin{aligned}
L W= & \left(1-\frac{1}{S}\right)[(1-q) A-\beta S I-(\mu+p) S+\gamma I+\varepsilon V]+\frac{1}{2} \sigma^{2} I^{2} \\
& +\left(1-\frac{1}{I}\right)[\beta S I-(\mu+\gamma+\alpha) I]+\frac{1}{2} \sigma^{2} S^{2}+\left(1-\frac{1}{V}\right)[q A+p S-(\mu+\varepsilon) V] \\
= & A-(\mu+p) S+\gamma I+\varepsilon V-(1-q) \frac{A}{S}+\beta I+(\mu+p)-\gamma \frac{I}{S}-\varepsilon \frac{V}{S} \\
& +\frac{1}{2} \sigma^{2} I^{2}-(\mu+\gamma+\alpha) I-\beta S+(\mu+\gamma+\alpha)+\frac{1}{2} \sigma^{2} S^{2}+p S \\
& -(\mu+\varepsilon) V-\frac{q A}{V}-\frac{p S}{V}+(\mu+\varepsilon) \\
\leq & A+\gamma I+\varepsilon V+\beta I+(\mu+p)+(\mu+\gamma+\alpha)+p S+(\mu+\varepsilon)+\frac{1}{2} \sigma^{2}\left(S^{2}+I^{2}\right) \\
\leq & A+(\gamma+\varepsilon+\beta+p) M+(3 \mu+p+\gamma+\alpha+\varepsilon)+\sigma^{2} M^{2}:=K .
\end{aligned}
$$

Moreover, substituting this into (2.2), we obtain

$$
d W(S, I, V) \leq K d t+\sigma(I-S) d B(t)
$$

We can now integrate both sides of (2.3) from 0 to $\tau_{k} \wedge T$ and then take the expectations,

$$
\begin{aligned}
E W\left(S\left(\tau_{k} \wedge T\right), I\left(\tau_{k} \wedge T\right), V\left(\tau_{k} \wedge T\right)\right) & \leq W(S(0), I(0), V(0))+K E\left(\tau_{k} \wedge T\right) \\
& \leq W(S(0), I(0), V(0))+K T
\end{aligned}
$$

Set $\Omega_{k}=\left\{\tau_{k} \leq T\right\}$ for $k \geq k_{1}$ and by (2.1), $P\left(\Omega_{k}\right) \geq \varepsilon$. Note that for every $\omega \in \Omega_{k}$, there is at least one of $S\left(\tau_{k}, \omega\right), I\left(\tau_{k}, \omega\right)$, and $V\left(\tau_{k}, \omega\right)$ that equals either $k$ or $1 / k$, and hence $W\left(S\left(\tau_{k}, \omega\right), I\left(\tau_{k}, \omega\right), V\left(\tau_{k}, \omega\right)\right)$ is not less than

$$
k-1-\log k \quad \text { or } \quad \frac{1}{k}-1-\log \frac{1}{k}=\frac{1}{k}-1+\log k .
$$

Consequently,

$$
W\left(S\left(\tau_{k}, \omega\right), I\left(\tau_{k}, \omega\right), V\left(\tau_{k}, \omega\right)\right) \geq[k-1-\log k] \wedge\left[\frac{1}{k}-1+\log k\right] .
$$

It then follows from (2.1) and (2.4) that

$$
\begin{aligned}
W(S(0), I(0), V(0))+K T & \geq E\left[1_{\Omega_{k}}(\omega) W\left(S\left(\tau_{k}, \omega\right), I\left(\tau_{k}, \omega\right), V\left(\tau_{k}, \omega\right)\right)\right] \\
& \geq \varepsilon\left[(k-1-\log k) \wedge\left(\frac{1}{k}-1+\log k\right)\right],
\end{aligned}
$$


where $1_{\Omega_{k}(\omega)}$ is the indicator function of $\Omega_{k}$. Letting $k \rightarrow \infty$ leads to the contradiction $\infty>W(S(0), I(0), V(0))+K T=\infty$. So we must therefore have $\tau_{\infty}=\infty$ a.s.

Remark 2.1 From Theorems 2.1 for any initial value $(S(0), I(0), V(0)) \in \mathbb{R}_{+}^{3}$, there is a unique global solution $(S(t), I(t), V(t)) \in \mathbb{R}_{+}^{3}$ almost surely of system (1.3). Hence

$$
d(S+I+V) \leq[A-\mu(S+I+V)] d t
$$

and

$$
S(t)+I(t)+V(t) \leq \frac{A}{\mu}+e^{-\mu t}\left(S(0)+I(0)+V(0)-\frac{A}{\mu}\right) .
$$

If $S(0)+I(0)+V(0) \leq A / \mu$, then $S(t)+I(t)+V(t) \leq A / \mu$ a.s. so the region

$$
\Gamma^{*}=\left\{(S, I, V): S>0, I>0, V>0, S+I+V \leq \frac{A}{\mu} \text { a.s. }\right\}
$$

is a positively invariant set of system (1.3) on $\Gamma^{*}$, which is similar to system (1.1).

From now on, we always assume that $(S(0), I(0), V(0)) \in \Gamma^{*}$.

\section{Exponential stability in large perturbation}

In this section, we show that the large perturbation forces the number of infected cases to zero exponentially regardless of the magnitude of $R_{0}$.

Theorem 3.1 Let $(S(t), I(t), V(t))$ be the solution of system (1.3) with initial value $(S(0), I(0)$, $V(0)) \in \Gamma^{*}$. If $\sigma^{2}>\beta^{2} / 2(\mu+\gamma+\alpha)$, then

$$
\limsup _{t \rightarrow \infty} \frac{\log I(t)}{t} \leq-(\mu+\gamma+\alpha)+\frac{\beta^{2}}{2 \sigma^{2}}<0 \quad \text { a.s. }
$$

Moreover,

$$
\begin{aligned}
& \lim _{t \rightarrow \infty} S(t)=\frac{A(\mu(1-q)+\varepsilon)}{\mu(\mu+\varepsilon+p)}=S_{0}, \\
& \lim _{t \rightarrow \infty} V(t)=\frac{A(\mu q+p)}{\mu(\mu+\varepsilon+p)}=V_{0} \quad \text { a.s. }
\end{aligned}
$$

(namely, $I(t)$ tends to zero exponentially a.s. i.e., the disease dies out with probability 1 ).

Proof Applying the Itô formula to system (1.3) leads to

$$
\begin{aligned}
d(\log I) & =\left[\beta S-(\mu+\gamma+\alpha)-\frac{1}{2} \sigma^{2} S^{2}\right] d t+\sigma S d B(t) \\
& \leq\left[-\frac{1}{2} \sigma^{2}\left(S-\frac{\beta}{\sigma^{2}}\right)^{2}-(\mu+\gamma+\alpha)+\frac{\beta^{2}}{2 \sigma^{2}}\right] d t+\sigma S d B(t) \\
& \leq\left[-(\mu+\gamma+\alpha)+\frac{\beta^{2}}{2 \sigma^{2}}\right] d t+\sigma S d B(t) .
\end{aligned}
$$


Integrating this from 0 to $t$ and dividing $t$ on the both sides, we have

$$
\frac{\log I(t)}{t} \leq-(\mu+\gamma+\alpha)+\frac{\beta^{2}}{2 \sigma^{2}}+\frac{M(t)}{t}+\frac{\log I(0)}{t}
$$

where $M(t):=\sigma \int_{0}^{t} S(r) d B(r)$, which is a local continuous martingale and $M(0)=0$. Moreover

$$
\limsup _{t \rightarrow \infty} \frac{\langle M, M\rangle_{t}}{t} \leq \sigma^{2}\left(\frac{A}{\mu}\right)^{2}<\infty \text { a.s. }
$$

By Lemma A.1 (in the Appendix), we obtain

$$
\lim _{t \rightarrow \infty} \frac{M(t)}{t}=0 \quad \text { a.s. }
$$

It follows from (3.2), taking the limit superior of both sides, that

$$
\limsup _{t \rightarrow \infty} \frac{\log I(t)}{t} \leq-(\mu+\gamma+\alpha)+\frac{\beta^{2}}{2 \sigma^{2}} \quad \text { a.s. }
$$

If $\sigma^{2}>\beta^{2} / 2(\mu+\gamma+\alpha)$, then

$$
\limsup _{t \rightarrow \infty} \frac{\log I(t)}{t}<0 \quad \text { a.s., }
$$

which implies

$$
\lim _{t \rightarrow \infty} I(t)=0 \quad \text { a.s. }
$$

Now we show that $V(t)$ and $S(t)$ converge a.s. to $V_{0}$ and $S_{0}$ as $t \rightarrow \infty$, respectively. According to system (1.3), we obtain

$$
d(S+I+V)=[A-\mu(S+I+V)-\alpha I] d t
$$

By (3.4) and (3.5), we have

$$
\lim _{t \rightarrow \infty}(S(t)+V(t))=\frac{A}{\mu} \quad \text { a.s. }
$$

According to (3.6), the last equation of the system (1.3) is an asymptotically differential system with limit system

$$
\dot{V}(t)=q A+p \frac{A}{\mu}-(\mu+\varepsilon+p) V(t)
$$

So we obtain

$$
\lim _{t \rightarrow \infty} V(t)=\frac{A(\mu q+p)}{\mu(\mu+\varepsilon+p)}=V_{0}
$$


Therefore, by (3.6), we have

$$
\lim _{t \rightarrow \infty} S(t)=\frac{A(\mu(1-q)+\varepsilon)}{\mu(\mu+\varepsilon+p)}=S_{0}
$$

This finishes the proof of Theorem 3.1.

Remark 3.1 When the perturbation is very large, the number of infected decays exponentially to zero. It makes sense in the point that the extinction of epidemics can be caused by the occurrence of a large perturbation.

\section{Asymptotic behavior around $P_{0}$ and $P^{*}$}

When studying epidemic dynamical systems, we are interested in two problems. One is when the disease will die out, and the other is when the disease will persist. In the section, we shall investigate the two problems according to the threshold $R_{0}$.

\subsection{Asymptotic behavior around $P_{0}$}

When $R_{0} \leq 1, P_{0}$ of system (1.1) is globally stable, which means the disease will die out. We shall show in Theorem 4.1 below, the same exponential stability of system (1.3), obtained in Theorem 3.1, continues valid when $R_{0}<1$. Moreover, in this case, we prove the solution to $(1.3)$ converges to $P_{0}$, a.s.

For convenience we introduce the following notation: Let

$$
\langle x(t)\rangle=\frac{1}{t} \int_{0}^{t} x(r) d B(r) .
$$

Theorem 4.1 Let $(S(t), I(t), V(t))$ be the solution of system $(1.3)$ with initial value $(S(0), I(0)$, $V(0)) \in \Gamma^{*}$. If $R_{0}<1$, then

$$
\limsup _{t \rightarrow \infty} \frac{\log I(t)}{t} \leq(\mu+\gamma+\alpha)\left(R_{0}-1\right)<0, \quad \text { a.s. }
$$

Moreover,

$$
\lim _{t \rightarrow \infty} S(t)=\frac{A(\mu(1-q)+\varepsilon)}{\mu(\mu+\varepsilon+p)}=S_{0}, \quad \lim _{t \rightarrow \infty} V(t)=\frac{A(\mu q+p)}{\mu(\mu+\varepsilon+p)}=V_{0} \quad \text { a.s. }
$$

Proof An integration of the system (1.3) yields

$$
\left\{\begin{aligned}
\frac{S(t)-S(0)}{t}= & (1-q) A-\beta\langle S(t) I(t)\rangle-(\mu+p)\langle S(t)\rangle+\gamma\langle I(t)\rangle+\varepsilon\langle V(t)\rangle \\
& -\frac{\sigma}{t} \int_{0}^{t} S(r) I(r) d B(r), \\
\frac{I(t)-I(0)}{t}= & \beta\langle S(t) I(t)\rangle-(\mu+\gamma+\alpha)\langle I(t)\rangle+\frac{\sigma}{t} \int_{0}^{t} S(r) I(r) d B(r), \\
\frac{V(t)-V(0)}{t}= & q A+p\langle S(t)\rangle-(\mu+\varepsilon)\langle V(t)\rangle .
\end{aligned}\right.
$$

According to (4.1), we have

$$
\begin{aligned}
& \frac{\mu+\varepsilon}{\mu}\left(\frac{S(t)-S(0)}{t}+\frac{I(t)-I(0)}{t}\right)+\frac{\varepsilon}{\mu} \frac{V(t)-V(0)}{t} \\
& =\frac{\mu(1-q)+\varepsilon}{\mu} A-(\mu+\varepsilon+p)\langle S(t)\rangle-\frac{(\mu+\varepsilon)(\mu+\alpha)}{\mu}\langle I(t)\rangle .
\end{aligned}
$$


We compute that

$$
\langle S(t)\rangle=\frac{1}{\mu+\varepsilon+p}\left(\frac{\mu(1-q)+\varepsilon}{\mu} A-\frac{(\mu+\varepsilon)(\mu+\alpha)}{\mu}\langle I(t)\rangle\right)+\varphi(t),
$$

where $\varphi(t)$ is defined by

$$
\varphi(t)=-\frac{1}{\mu+\varepsilon+p}\left[\frac{\mu+\varepsilon}{\mu}\left(\frac{S(t)-S(0)}{t}+\frac{I(t)-I(0)}{t}\right)+\frac{\varepsilon}{\mu}\left(\frac{V(t)-V(0)}{t}\right)\right] .
$$

Obviously $\varphi(t) \rightarrow 0, t \rightarrow \infty$. Substituting (4.2) into the first equation of (3.1), we have

$$
\begin{aligned}
\frac{\log I(t)-\log I(0)}{t}= & \beta\langle S(t)\rangle-(\mu+\gamma+\alpha)-\frac{1}{2} \sigma^{2}\left\langle S^{2}(t)\right\rangle+\frac{\sigma}{t} \int_{0}^{t} S(r) d B(r) \\
= & \beta \frac{A}{\mu} \frac{\mu(1-q)+\varepsilon}{\mu+\varepsilon+p}-(\mu+\gamma+\alpha)-\frac{1}{2} \sigma^{2}\left\langle S^{2}(t)\right\rangle \\
& -\frac{\beta(\mu+\varepsilon)(\mu+\alpha)}{\mu(\mu+\varepsilon+p)}\langle I(t)\rangle+\beta \varphi(t)+\frac{\sigma}{t} \int_{0}^{t} S(r) d B(r) \\
= & (\mu+\gamma+\alpha)\left(R_{0}-1\right)-\frac{1}{2} \sigma^{2}\left\langle S^{2}(t)\right\rangle-\frac{\beta(\mu+\varepsilon)(\mu+\alpha)}{\mu(\mu+\varepsilon+p)}\langle I(t)\rangle \\
& +\beta \varphi(t)+\frac{\sigma}{t} \int_{0}^{t} S(r) d B(r) \\
\leq & (\mu+\gamma+\alpha)\left(R_{0}-1\right)+\beta \varphi(t)+\frac{\sigma}{t} \int_{0}^{t} S(r) d B(r)
\end{aligned}
$$

and hence

$$
\frac{\log I(t)}{t} \leq(\mu+\gamma+\alpha)\left(R_{0}-1\right)+\beta \varphi(t)+\frac{M(t)}{t}+\frac{\log I(0)}{t},
$$

where $M(t):=\sigma \int_{0}^{t} S(r) d B(r)$. By (3.3) and Lemma A.1 (in the Appendix), we obtain $\lim _{t \rightarrow \infty} \frac{M(t)}{t}=0$ a.s. Taking the limit superior of both sides of (4.3), if $R_{0}<1$, we get

$$
\limsup _{t \rightarrow \infty} \frac{\log I(t)}{t} \leq(\mu+\gamma+\alpha)\left(R_{0}-1\right)<0 \quad \text { a.s. }
$$

The remainder of the proof follows that in Theorem 3.1, so we have

$$
\begin{aligned}
& \lim _{t \rightarrow \infty} S(t)=\frac{A(\mu(1-q)+\varepsilon)}{\mu(\mu+\varepsilon+p)}=S_{0}, \\
& \lim _{t \rightarrow \infty} V(t)=\frac{A(\mu q+p)}{\mu(\mu+\varepsilon+p)}=V_{0} \quad \text { a.s. }
\end{aligned}
$$

This finishes the proof of Theorem 4.1.

\subsection{Asymptotic behavior around $P^{*}$}

In the deterministic models, the second problem is usually solved by showing that the endemic equilibrium to the corresponding model is a global attractor or is globally asymptotically stable under some conditions. But there is no endemic equilibrium of system 
(1.3). How can one measure whether the disease will persist? In this section, we show that the difference between the solution of system (1.3) and $P^{*}$ is small if white noise is weak, reflecting that the disease is prevalent.

Theorem 4.2 Assume $R_{0}>1$. Let $(S(t), I(t), V(t))$ be the solution of system (1.3) with initial value $(S(0), I(0), V(0)) \in \Gamma^{*}$. If $\alpha^{2}<4 \mu(\mu+\alpha)(1+2 \mu / \varepsilon)$, then

$$
\begin{aligned}
\limsup _{t \rightarrow \infty} & \frac{1}{t} \int_{0}^{t}\left[\mu\left(\frac{2(\mu+p)}{\varepsilon}+1\right)\left(S(r)-S^{*}\right)^{2}+\left((\mu+\alpha)\left(1+\frac{2 \mu}{\varepsilon}\right)-\frac{\alpha}{4 \rho}\right)\left(I(r)-I^{*}\right)^{2}\right. \\
& \left.+(\mu-\rho \alpha)\left(V(r)-V^{*}\right)^{2}\right] d r \\
\leq & \frac{1}{2 \beta}\left(\frac{2 \mu}{\varepsilon}(2 \mu+p+\alpha)+(2 \mu+\alpha)\right)\left(\frac{A}{\mu}\right)^{2} I^{*} \sigma^{2} \quad \text { a.s. }
\end{aligned}
$$

where $P^{*}=\left(S^{*}, I^{*}, V^{*}\right)$ is the endemic equilibrium of system (1.1), $\rho$ is positive constant satisfied $\alpha / 4(\mu+\alpha)(1+2 \mu / \varepsilon)<\rho<\mu / \alpha$.

Proof Since $P^{*}$ is the endemic equilibrium of system (1.1), we have

$$
\begin{aligned}
& (1-q) A=\beta S^{*} I^{*}-(\mu+p) S^{*}+\gamma I^{*}+\varepsilon V^{*}, \quad \beta S^{*} I^{*}=(\mu+\gamma+\alpha) I^{*}, \\
& q A+p S^{*}=(\mu+\varepsilon) V^{*} .
\end{aligned}
$$

Define a $C^{2}$-function $W: \mathbb{R}_{+}^{3} \rightarrow \mathbb{R}_{+}$by

$$
\begin{aligned}
W(S, I, V)= & c_{1}\left(I-I^{*}-I^{*} \log \frac{I}{I^{*}}\right)+\frac{c_{2}}{2}\left[\left(S-S^{*}\right)+\left(I-I^{*}\right)\right]^{2} \\
& +\frac{1}{2}\left[\left(S-S^{*}\right)+\left(I-I^{*}\right)+\left(V-V^{*}\right)\right]^{2} \\
:= & c_{1} W_{1}+c_{2} W_{2}+W_{3},
\end{aligned}
$$

where $c_{1}$ and $c_{2}$ are positive constants to be determined later. Let $L$ be the generating operator of system (1.3). Then we get

$$
\begin{aligned}
L W_{1}= & \beta\left(S-S^{*}\right)\left(I-I^{*}\right)+\frac{1}{2} \sigma^{2} S^{2} I^{*} \\
L W_{2}= & {\left[\left(S-S^{*}\right)+\left(I-I^{*}\right)\right]\left[-(\mu+p)\left(S-S^{*}\right)-(\mu+\alpha)\left(I-I^{*}\right)+\varepsilon\left(V-V^{*}\right)\right] } \\
= & -(\mu+p)\left(S-S^{*}\right)^{2}-(\mu+\alpha)\left(I-I^{*}\right)^{2}-[(\mu+p)+(\mu+\alpha)]\left(S-S^{*}\right)\left(I-I^{*}\right) \\
& +\varepsilon\left(S-S^{*}\right)\left(V-V^{*}\right)+\varepsilon\left(I-I^{*}\right)\left(V-V^{*}\right)
\end{aligned}
$$

and

$$
\begin{aligned}
L W_{3}= & {\left[\left(S-S^{*}\right)+\left(I-I^{*}\right)+\left(V-V^{*}\right)\right]\left[-\mu\left(S-S^{*}\right)-(\mu+\alpha)\left(I-I^{*}\right)-\mu\left(V-V^{*}\right)\right] } \\
= & -\mu\left(S-S^{*}\right)^{2}-(\mu+\alpha)\left(I-I^{*}\right)^{2}-\mu\left(V-V^{*}\right)^{2}-(2 \mu+\alpha)\left(S-S^{*}\right)\left(I-I^{*}\right) \\
& -2 \mu\left(S-S^{*}\right)\left(V-V^{*}\right)-(2 \mu+\alpha)\left(I-I^{*}\right)\left(V-V^{*}\right),
\end{aligned}
$$


where (4.5) is used. Therefore

$$
\begin{aligned}
L W= & {\left[c_{1} \beta-c_{2}(2 \mu+p+\alpha)-(2 \mu+\alpha)\right]\left(S-S^{*}\right)\left(I-I^{*}\right)-\left[c_{2}(\mu+p)+\mu\right]\left(S-S^{*}\right)^{2} } \\
& -\left(c_{2}+1\right)(\mu+\alpha)\left(I-I^{*}\right)^{2}-\mu\left(V-V^{*}\right)^{2}+\left(c_{2} \varepsilon-2 \mu\right)\left(S-S^{*}\right)\left(V-V^{*}\right) \\
& +\left[c_{2} \varepsilon-(2 \mu+\alpha)\right]\left(I-I^{*}\right)\left(V-V^{*}\right)+\frac{c_{1}}{2} \sigma^{2} S^{2} I^{*} .
\end{aligned}
$$

Choose $c_{1}=(2 \mu(2 \mu+p+\alpha) / \varepsilon+(2 \mu+\alpha)) / \beta$ and $c_{2}=2 \mu / \varepsilon$ such that

$$
c_{1} \beta-c_{2}(2 \mu+p+\alpha)-(2 \mu+\alpha)=0, c_{2} \varepsilon-2 \mu=0 .
$$

Then

$$
\begin{aligned}
L W= & -\mu\left[\frac{2(\mu+p)}{\varepsilon}+1\right]\left(S-S^{*}\right)^{2}-\left(1+\frac{2 \mu}{\varepsilon}\right)(\mu+\alpha)\left(I-I^{*}\right)^{2} \\
& -\mu\left(V-V^{*}\right)^{2}-\alpha\left(I-I^{*}\right)\left(V-V^{*}\right)+\frac{c_{1}}{2} \sigma^{2} S^{2} I^{*} \\
\leq & -\mu\left[\frac{2(\mu+p)}{\varepsilon}+1\right]\left(S-S^{*}\right)^{2}-\left[(\mu+\alpha)\left(1+\frac{2 \mu}{\varepsilon}\right)-\frac{\alpha}{4 \rho}\right]\left(I-I^{*}\right)^{2} \\
& -(\mu-\rho \alpha)\left(V-V^{*}\right)^{2}+\frac{c_{1}}{2}\left(\frac{A}{\mu}\right)^{2} I^{*} \sigma^{2} \\
:= & F(t),
\end{aligned}
$$

where $\rho$ is positive constant to be specified later and Young's inequality is used. If

$$
\alpha^{2}<4 \mu(\mu+\alpha)\left(1+\frac{2 \mu}{\varepsilon}\right)
$$

we choose $\rho$ such that $\alpha / 4(\mu+\alpha)(1+2 \mu / \varepsilon)<\rho<\mu / \alpha$. This implies

$$
(\mu+\alpha)\left(1+\frac{2 \mu}{\varepsilon}\right)-\frac{\alpha}{4 \rho}>0 \quad \text { and } \quad \mu-\rho \alpha>0 .
$$

Therefore,

$$
d W \leq F(t) d t+\sigma S\left(I-I^{*}\right) d B(t)
$$

Integrating both sides from 0 to $t$ yields

$$
W_{2}(t)-W_{2}(0) \leq \int_{0}^{t} F(s) d s+\int_{0}^{t} \sigma S\left(I-I^{*}\right) d B(s)
$$

Let $M(t):=\int_{0}^{t} \sigma S\left(I-I^{*}\right) d B(s)$, which is a local continuous martingale and $M(0)=0$. Moreover,

$$
\limsup _{t \rightarrow \infty} \frac{\langle M, M\rangle_{t}}{t} \leq 4 \sigma^{2}\left(\frac{A}{\mu}\right)^{4}<\infty
$$


By Lemma A.1 (in the Appendix), we obtain

$$
\lim _{t \rightarrow \infty} \frac{M(t)}{t}=0 \quad \text { a.s., }
$$

which together with (4.6) implies

$$
\liminf _{t \rightarrow \infty} \frac{\int_{0}^{t} F(s) d s}{t} \geq 0 \quad \text { a.s. }
$$

Consequently,

$$
\begin{aligned}
\limsup _{t \rightarrow \infty} & \frac{1}{t} \int_{0}^{t}\left[\mu\left(\frac{2(\mu+p)}{\varepsilon}+1\right)\left(S(r)-S^{*}\right)^{2}+\left((\mu+\alpha)\left(1+\frac{2 \mu}{\varepsilon}\right)-\frac{\alpha}{4 \rho}\right)\left(I(r)-I^{*}\right)^{2}\right. \\
& \left.+(\mu-\rho \alpha)\left(V(r)-V^{*}\right)^{2}\right] d r \\
\leq & \frac{1}{2 \beta}\left(\frac{2 \mu}{\varepsilon}(2 \mu+p+\alpha)+(2 \mu+\alpha)\right)\left(\frac{A}{\mu}\right)^{2} I^{*} \sigma^{2} \quad \text { a.s. }
\end{aligned}
$$

Thus, Theorem 4.2 is proved.

Remark 4.1 Theorem 4.2 shows that, under some conditions, the distance between the solution $X(t)=(S(t), I(t), V(t))$ and the endemic equilibrium $P^{*}=\left(S^{*}, I^{*}, V^{*}\right)$ of system (1.1) has the following form:

$$
\limsup _{t \rightarrow \infty} \frac{1}{t} \int_{0}^{t}\left\|X(s)-P^{*}\right\|^{2} d s \leq C\|\sigma\|^{2} \text {, a.s., }
$$

where $C$ is a positive constant. Although the solution of system (1.3) does not have stability as the deterministic system, we can think there is approximate stability, provided $\|\sigma\|^{2}$ is sufficiently small. In this situation, we consider the disease to persist.

From the result of Theorem 4.2, we conclude system (1.3) is persistent, which also reflects that the disease is prevalent. Chen et al. in [21] proposed the definition of persistence in the mean for the deterministic system. Here, we also use this definition for the stochastic system.

Definition 4.1 System (1.3) is said to be persistent in the mean, if

$$
\begin{aligned}
& \liminf _{t \rightarrow \infty} \frac{1}{t} \int_{0}^{t} S(r) d r>0, \quad \liminf _{t \rightarrow \infty} \frac{1}{t} \int_{0}^{t} I(r) d r>0, \\
& \liminf _{t \rightarrow \infty} \frac{1}{t} \int_{0}^{t} V(r) d r>0, \quad \text { a.s. }
\end{aligned}
$$

Theorem 4.3 Assume $R_{0}>1$. Let $(S(t), I(t), V(t))$ be the solution of system (1.3) with initial value $(S(0), I(0), V(0)) \in \Gamma^{*}$. If $\alpha^{2}<4 \mu(\mu+\alpha)(1+2 \mu / \varepsilon)$ and

$$
\begin{aligned}
& \min \left\{\mu\left(\frac{2(\mu+p)}{\varepsilon}+1\right)\left(S^{*}\right)^{2},\left((\mu+\alpha)\left(1+\frac{2 \mu}{\varepsilon}\right)-\frac{\alpha}{4 \rho}\right)\left(I^{*}\right)^{2},(\mu-\rho \alpha)\left(V^{*}\right)^{2}\right\} \\
& >\frac{1}{2 \beta}\left(\frac{2 \mu}{\varepsilon}(2 \mu+p+\alpha)+(2 \mu+\alpha)\right)\left(\frac{A}{\mu}\right)^{2} I^{*} \sigma^{2},
\end{aligned}
$$


where $\rho$ is a positive constant satisfying $\alpha / 4(\mu+\alpha)(1+2 \mu / \varepsilon)<\rho<\mu / \alpha$, then system (1.3) is persistent in the mean.

Proof Obviously, (4.4) is true. That is to say, we have

$$
\begin{aligned}
& \limsup _{t \rightarrow \infty} \frac{1}{t} \int_{0}^{t} \mu\left(\frac{2(\mu+p)}{\varepsilon}+1\right)\left(S-S^{*}\right)^{2} d r \\
& \quad \leq \frac{1}{2 \beta}\left(\frac{2 \mu}{\varepsilon}(2 \mu+p+\alpha)+(2 \mu+\alpha)\right)\left(\frac{A}{\mu}\right)^{2} I^{*} \sigma^{2} \quad \text { a.s., } \\
& \limsup _{t \rightarrow \infty} \frac{1}{t} \int_{0}^{t}\left((\mu+\alpha)\left(1+\frac{2 \mu}{\varepsilon}\right)-\frac{\alpha}{4 \rho}\right)\left(I-I^{*}\right)^{2} d r \\
& \quad \leq \frac{1}{2 \beta}\left(\frac{2 \mu}{\varepsilon}(2 \mu+p+\alpha)+(2 \mu+\alpha)\right)\left(\frac{A}{\mu}\right)^{2} I^{*} \sigma^{2} \quad \text { a.s., } \\
& \limsup _{t \rightarrow \infty} \frac{1}{t} \int_{0}^{t}(\mu-\rho \alpha)\left(V-V^{*}\right)^{2} d r \\
& \quad \leq \frac{1}{2 \beta}\left(\frac{2 \mu}{\varepsilon}(2 \mu+p+\alpha)+(2 \mu+\alpha)\right)\left(\frac{A}{\mu}\right)^{2} I^{*} \sigma^{2} \quad \text { a.s. }
\end{aligned}
$$

Besides,

$$
\left(S^{*}\right)^{2}+\left(S-S^{*}\right)^{2} \geq 2 S^{*}\left(S^{*}-S\right)
$$

i.e.

$$
S \geq \frac{S^{*}}{2}-\frac{\left(S-S^{*}\right)^{2}}{2 S^{*}}
$$

Together with (4.7) and (4.8), we get

$$
\begin{aligned}
\liminf _{t \rightarrow \infty} \frac{1}{t} \int_{0}^{t} S(r) d r & \geq \frac{S^{*}}{2}-\limsup _{t \rightarrow \infty} \frac{1}{t} \int_{0}^{t} \frac{\left(S-S^{*}\right)^{2}}{2 S^{*}} d r \\
& \geq \frac{S^{*}}{2}-\frac{1}{4 S^{*}} \frac{\left(\frac{2 \mu}{\varepsilon}(2 \mu+p+\alpha)+(2 \mu+\alpha)\right)\left(\frac{A}{\mu}\right)^{2} I^{*} \sigma^{2}}{\beta \mu\left(\frac{2(\mu+p)}{\varepsilon}+1\right)}>0 \text { a.s. }
\end{aligned}
$$

Similarly,

$$
\liminf _{t \rightarrow \infty} \frac{1}{t} \int_{0}^{t} I(s) d s \geq \frac{I^{*}}{2}-\frac{1}{4} \frac{\left(\frac{2 \mu}{\varepsilon}(2 \mu+p+\alpha)+(2 \mu+\alpha)\right)\left(\frac{A}{\mu}\right)^{2} \sigma^{2}}{\beta\left((\mu+\alpha)\left(1+\frac{2 \mu}{\varepsilon}\right)-\frac{\alpha}{4 \rho}\right)}>0 \quad \text { a.s. }
$$

and

$$
\liminf _{t \rightarrow \infty} \frac{1}{t} \int_{0}^{t} V(s) d s \geq \frac{V^{*}}{2}-\frac{1}{4 V^{*}} \frac{\left(\frac{2 \mu}{\varepsilon}(2 \mu+p+\alpha)+(2 \mu+\alpha)\right)\left(\frac{A}{\mu}\right)^{2} I^{*} \sigma^{2}}{\beta(\mu-\rho \alpha)}>0 \quad \text { a.s. }
$$

Consequently, system (1.3) is persistent in the mean.

Remark 4.2 When the disease-related death rate is small, Theorem 4.3 shows that if the perturbation is so small that it satisfies the condition (4.7), system (1.3) is persistent in the mean. 


\section{Numerical simulations}

In order to conform the results above, we numerically simulate the solution of system (1.3) with the initial value $(S(0), I(0), V(0))=(0.8,0.4,0.5)$. Using Milstein's higher order method mentioned in [22], we get the discretization equation of system (1.3):

$$
\left\{\begin{aligned}
S_{k+1}= & S_{k}+\left((1-q) A-\beta S_{k} I_{k}-(\mu+p) S_{k}+\gamma I_{k}+\varepsilon V_{k}\right) \Delta t-\sigma S_{k} I_{k} \sqrt{\Delta t} \xi_{k} \\
& +\frac{\sigma^{2}}{2} S_{k} I_{k}^{2} \Delta t\left(\xi_{k}^{2}-1\right), \\
I_{k+1}= & I_{k}+\left(\beta S_{k} I_{k}-(\mu+\gamma+\alpha) I_{k}\right) \Delta t+\sigma S_{k} I_{k} \sqrt{\Delta t} \xi_{k}+\frac{\sigma^{2}}{2} S_{k}^{2} I_{k} \Delta t\left(\xi_{k}^{2}-1\right), \\
V_{k+1}= & V_{k}+\left(q A+p S_{k}-(\mu+\varepsilon) V_{k}\right) \Delta t,
\end{aligned}\right.
$$

where $\xi_{k}, k=1,2, \ldots, n$, are the independent Gaussian random variables $N(0,1)$.

By Theorem 3.1, we expect that the disease-free equilibrium $P_{0}$ of system (1.3) is exponentially stable under $\sigma^{2}>\beta^{2} / 2(\mu+\gamma+\alpha)$. In Figure 1, we choose parameters $A=0.9$; $q=0.1 ; \beta=0.8 ; \mu=0.4 ; p=0.1 ; \gamma=0.1 ; \varepsilon=0.2 ; \alpha=0.1 ; \sigma=0.9$, such that $R_{0}=2.4>1$ and $\sigma^{2}=0.81>8 / 15=\beta^{2} / 2(\mu+\gamma+\alpha)$. Then the disease-free equilibrium $P_{0}$ of system (1.3) is exponentially stable. But when $R_{0}>1$, there is an endemic equilibrium $P^{*}$ of system (1.1), and it is globally asymptotically stable under the sufficient condition $(\mu+\varepsilon)(2 \mu+\alpha)^{2}=$ $0.486>0.007=\alpha^{2}(\mu+\varepsilon+p)$ (see Li and Ma [7]). Figure 1 illustrates this visually.

By Theorem 4.1, we expect that the disease-free equilibrium $P_{0}$ of system (1.3) is exponentially stable under $R_{0}<1$. In Figure 2 , we choose parameters $A=0.2 ; q=0.5 ; \beta=0.5$; $\mu=0.2 ; p=0.3 ; \gamma=0.2 ; \varepsilon=0.1 ; \alpha=0.3 ; \sigma=0.3$, such that $R_{0}=5 / 21<1$. Then the diseasefree equilibrium $P_{0}$ of systems (1.3) is exponentially stable and the disease-free equilibrium $P_{0}$ of system (1.1) is globally asymptotically stable. Figure 2 illustrates this visually.
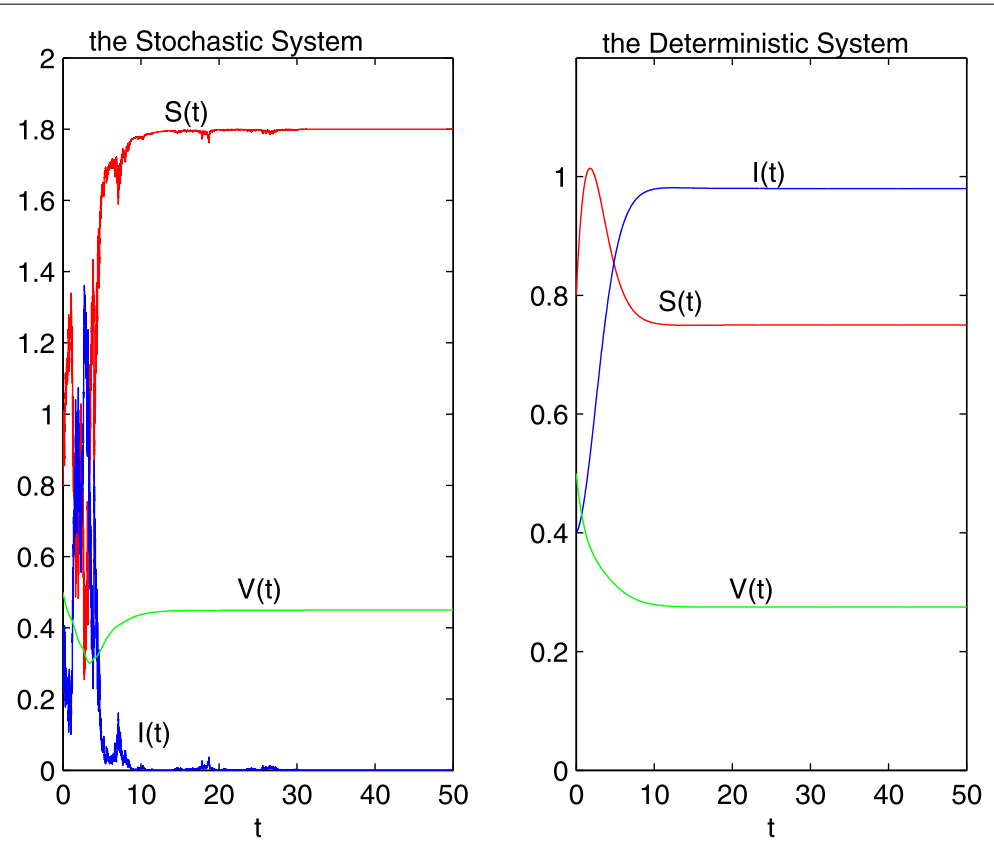

Figure 1 The solution of system (1.3) and system (1.1) with $\Delta t=0.001, R_{0}>1$. The picture on the left represents the solution of system (1.3) with $\sigma=0.9$, such that $\sigma^{2}>\beta^{2} / 2(\mu+\gamma+\alpha)$ and the other represents the solution of the corresponding undisturbed system (1.1). 

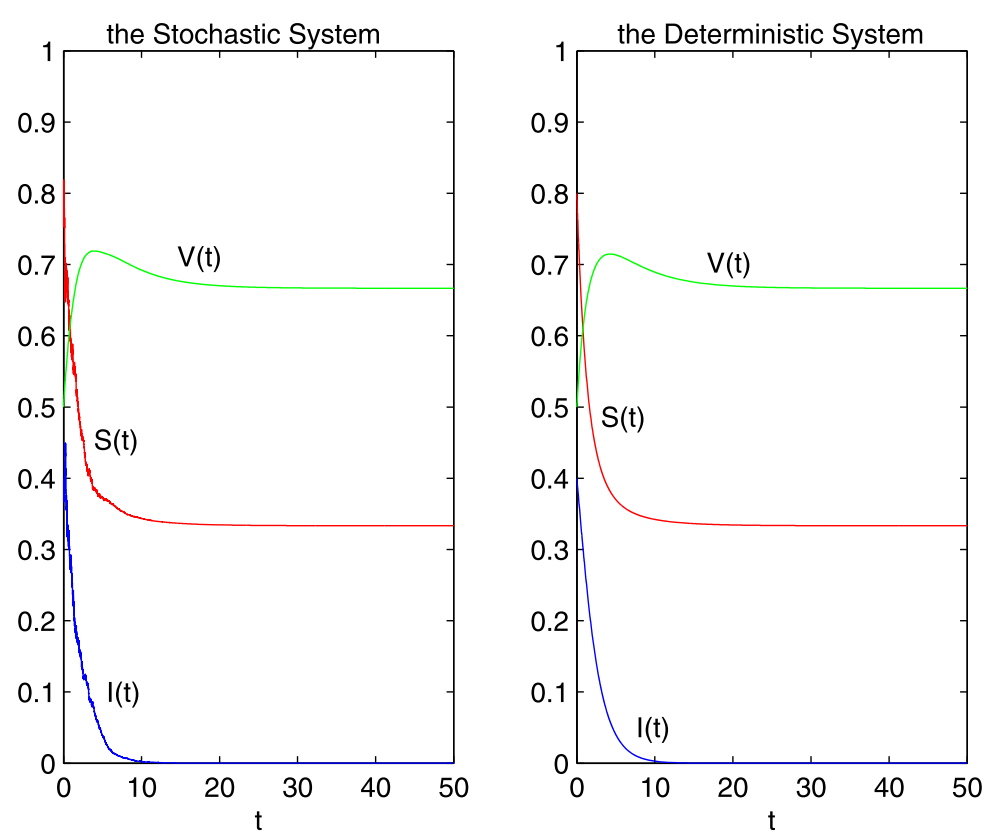

Figure 2 The solution of system (1.3) and system (1.1) with $\Delta t=0.001, R_{0}<1$. The picture on the left represents the solution of system (1.3) with $\sigma=0.3$, and the other represents the solution of the corresponding undisturbed system (1.1).

Next, we consider the effect of stochastic fluctuations of environment to the endemic equilibrium of the corresponding deterministic system. Theorem 4.2 tells us that the difference between the perturbed solution and $P^{*}$ is only related with white noise under the condition $R_{0}>1$ and $\alpha^{2}<4 \mu(\mu+\alpha)(1+2 \mu / \varepsilon)$. In Figure 3, we choose the same parameters as in Figure 1 except for the sigma values $\sigma=0.03$, such that $R_{0}=87 / 35>1$ and $\alpha^{2}=0.01<4=4 \mu(\mu+\alpha)(1+2 \mu / \varepsilon)$. As expected, the solution is oscillating around the endemic equilibrium $P^{*}$ for a long time (see Figure 3 ). Besides, the parameters of the middle plot in Figure 3 are the same but with different white noise $\sigma=0.01$. Obviously, we can observe that, with white noise getting weaker, the fluctuation around $P^{*}$ gets smaller, which supports the result of Theorem 4.2.

Finally, we keep the system parameters the same as in Figure 3 but we let $\sigma=0.04$. It is easy to verify that the system parameters obey the condition (4.7), $\sigma^{2}=0.0016<$ $l \min \left\{\mu(2(\mu+p) / \varepsilon+1)\left(S^{*}\right)^{2},((\mu+\alpha)(1+2 \mu / \varepsilon)-\alpha / 4 \rho)\left(I^{*}\right)^{2},(\mu-\rho \alpha)\left(V^{*}\right)^{2}\right\} \approx 0.0019$, where $l=2 \beta /(2 \mu / \varepsilon(2 \mu+p+\alpha)+(2 \mu+\alpha))(A / \mu)^{2} I^{*}=0.0658$. We can therefore conclude system (1.3) is persistent in the mean. The computer simulation shown in Figure 4 clearly supports this result.

\section{Conclusions}

This paper studies the extinction and persistence in the mean of a stochastic SIS epidemic model with vaccination. When the perturbation is very large, the number of infected decays exponentially to zero, which means the disease will die out. A similar conclusion to the system (1.2) is obtained in Theorem 4.3 in [12], but there one simplified system (1.2) into a single equation without vaccination and $\alpha=0$. The present paper is the first attempt, to the best of our knowledge, of such a study of a large perturbation that surpasses 

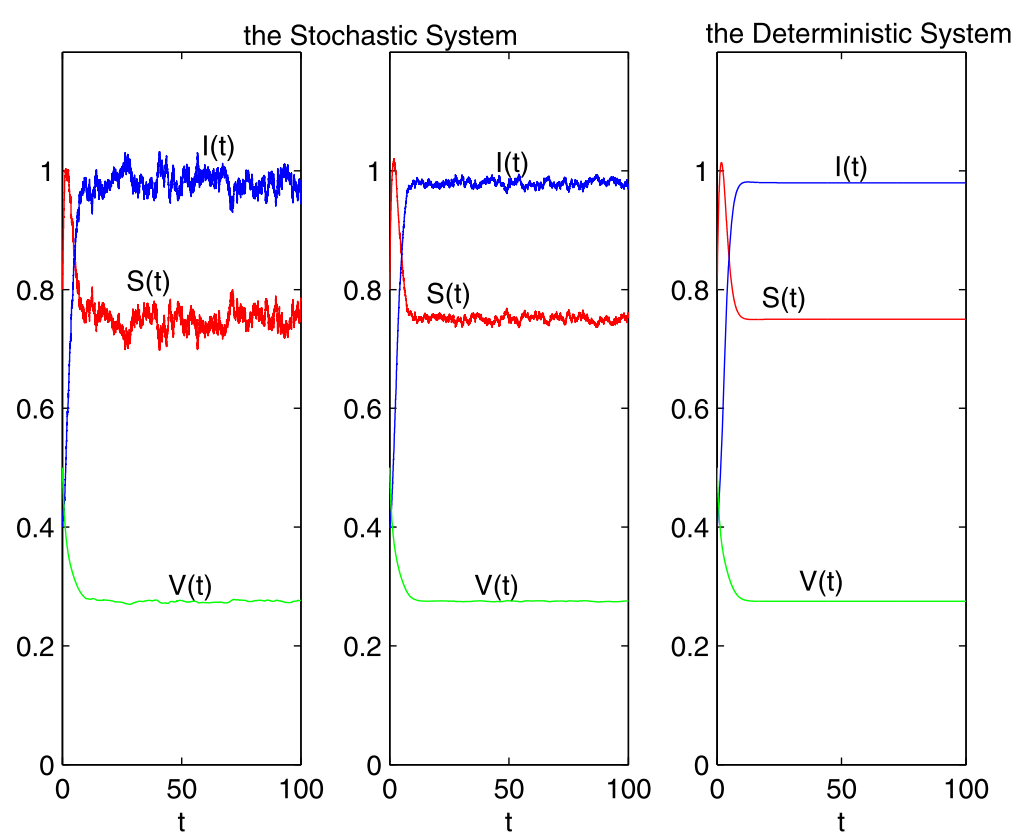

Figure 3 The solution of system (1.3) and system (1.1) with $\Delta t=0.001, R_{0}>1$. The first two pictures on the left represents the solution of system (1.3) with $\sigma=0.03$ and $\sigma=0.01$, respectively, and the other represents the solution of the corresponding undisturbed system (1.1).

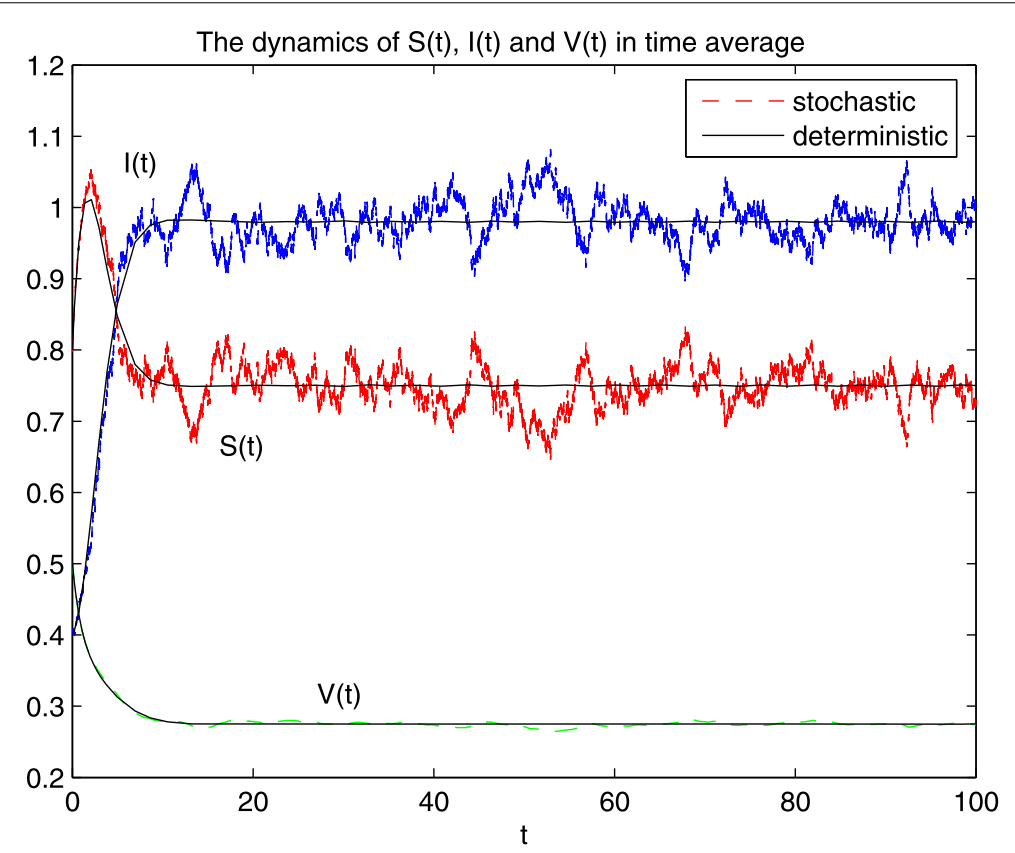

Figure 4 Computer simulation of the path $S(t), I(t)$, and $V(t)$ of the SDE model (1.3) for $\sigma=0.04$, using the EM method with step size $\Delta t=0.001$ and the initial value $(S(0), I(0), V(0))=(0.8,0.4,0.5)$.

the effect of $R_{0}$ as a threshold value in a high-dimensional system. Theorem 4.1 showed $P_{0}$ is exponentially stable if $R_{0}<1$. The result is better than the asymptotic stability of $P_{0}$ in Theorem 3.1 in [11]. From Theorems 4.2 and 4.3, when the perturbation and the disease- 
related death rate are small, if $R_{0}>1$, we see that the disease will persist in the mean. In such a case, $R_{0}$ plays a role similar to the threshold of the deterministic model. Hence, a large perturbation surpasses the effect of $R_{0}$ as a threshold value, and a small perturbation retains some role of $R_{0}$ in a stochastic sense. Also, it is interesting to study the threshold $\tilde{R}_{0}$ of a stochastic SIR model, and these investigations are in progress.

\section{Appendix}

Next, we give some basic theory in stochastic differential equations (see [20]).

Throughout this paper, unless otherwise specified, let $\left(\Omega,\left\{\mathcal{F}_{t}\right\}_{t \geq 0}, P\right)$ be a complete probability space with a filtration $\left\{\mathcal{F}_{t}\right\}_{t \geq 0}$ satisfying the usual conditions (i.e. it is right continuous and $\mathcal{F}_{0}$ contains all $P$-null sets). Let

$$
\mathbb{R}_{+}^{d}=\left\{x \in \mathbb{R}^{d}: x_{i}>0 \text { for all } 1 \leq i \leq d\right\}, \quad \overline{\mathbb{R}}_{+}^{d}=\left\{x \in \mathbb{R}^{d}: x_{i} \geq 0 \text { for all } 1 \leq i \leq d\right\}
$$

In general, consider the $n$-dimensional stochastic differential equation

$$
d x(t)=f(x(t), t) d t+g(x(t), t) d B(t), \quad \text { for } t \geq t_{0},
$$

with initial value $x\left(t_{0}\right)=x_{0} \in R^{d}$. $B(t)$ denotes the $n$ dimensional standard Brownian motion defined on the above probability space. Define the differential operator $L$ associated with (A.1) by

$$
L=\frac{\partial}{\partial t}+\sum_{i=1}^{d} f_{i}(x, t) \frac{\partial}{\partial x_{i}}+\frac{1}{2} \sum_{i, j=1}^{d}\left[g^{T}(x, t) g(x, t)\right]_{i j} \frac{\partial^{2}}{\partial x_{i} \partial x_{j}} .
$$

If $L$ acts on a function $V \in C^{2,1}\left(\mathbb{R}^{d} \times \overline{\mathbb{R}}_{+} ; \overline{\mathbb{R}}_{+}\right)$, then

$$
L V(x, t)=V_{t}(x, t)+V_{x}(x, t) f(x, t)+\frac{1}{2} \operatorname{trace}\left[g^{T}(x, t) V_{x x}(x, t) g(x, t)\right],
$$

where $V_{t}=\frac{\partial V}{\partial t}, V_{x}=\left(\frac{\partial V}{\partial x_{1}}, \ldots, \frac{\partial V}{\partial x_{d}}\right), V_{x x}=\left(\frac{\partial^{2} V}{\partial x_{i} \partial x_{j}}\right) d \times d$. By Itô's formula, if $x(t) \in R^{d}$, then

$$
d V(x(t), t)=L V(x(t), t) d t+V_{x}(x(t), t) g(x(t), t) d B(t) .
$$

Consider (A.1), assume $f(0, t)=0$ and $g(0, t)=0$ for all $t \geq t_{0}$. So $x(t) \equiv 0$ is a solution of (A.1), called the trivial solution or the equilibrium position.

Let $D_{n}=(-n, n)$ for $n=1,2, \ldots . \tau_{n}=\tau_{D_{n}}$ be the first time the process has absolute value $n$. Since a diffusion process is continuous, it must reach level $n$ before it reaches level $n+1$. Therefore $\tau_{n}$ are non-decreasing, hence they converge to a limit $\tau_{\infty}=\lim _{n \rightarrow \infty} \tau_{n}$. Explosion occurs on the set $\left\{\tau_{\infty}<\infty\right\}$, because on this set, by continuity of $x(t), x\left(\tau_{\infty}\right)=$ $\lim _{n \rightarrow \infty} x\left(\tau_{n}\right)$. Thus $\left|x\left(\tau_{\infty}\right)\right|=\lim _{n \rightarrow \infty}\left|x\left(\tau_{n}\right)\right|=\lim _{n \rightarrow \infty} n=\infty$, and infinity is reached in a finite time on this set.

Definition A.1 Diffusion started from $x$ explodes if $P_{x}\left(\tau_{\infty}<\infty\right)>0$.

Definition A.2 A random variable $\tau: \Omega \rightarrow[0, \infty]$ (it may take the value $\infty$ ) is called an $\left\{\mathcal{F}_{t}\right\}$-stopping time (or simply, stopping time) if $\{\omega: \tau(\omega) \leq t\} \in \mathcal{F}_{t}$ for any $t \geq 0$. 
Lemma A.1 (Strong Law of Large Numbers) Let $M=\left\{M_{t}\right\}_{t \geq 0}$ be a real-value continuous local martingale vanishing at $t=0$. Then

$$
\lim _{t \rightarrow \infty}\langle M, M\rangle_{t}=\infty \quad \text { a.s. } \Rightarrow \lim _{t \rightarrow \infty} \frac{M_{t}}{\langle M, M\rangle_{t}}=0 \quad \text { a.s. }
$$

and also

$$
\limsup _{t \rightarrow \infty} \frac{\langle M, M\rangle_{t}}{t}<\infty \quad \text { a.s. } \Rightarrow \lim _{t \rightarrow \infty} \frac{M_{t}}{t}=0 \quad \text { a.s. }
$$

\section{Competing interests}

The authors declare that they have no competing interests.

\section{Authors' contributions}

The authors have contributed to the manuscript on an equal basis. All authors read and approved the final manuscript.

\section{Author details}

${ }^{1}$ School of Mathematics and Statistics, Northeast Normal University, Changchun, Jilin 130024, P. R. China. ${ }^{2}$ School of Science, Changchun University, Changchun, Jilin 130022, P. R. China. ${ }^{3}$ College of Science, China University of Petroleum (East China), Qingdao, 266580, China.

\section{Acknowledgements}

The work was supported by Program for NSFC of China (No: 11371085,11426060), and the Fundamental Research Funds for the Central Universities (No: 15CX08011A), the Scientific and Technological Research Project of Jilin Province's Education Department (No: 2012244) and the Education Science Research Project of Jilin Province (No: GH150104).

\section{Received: 5 January 2015 Accepted: 3 August 2015 Published online: 22 October 2015}

\section{References}

1. Chen, FH: A susceptible-infected epidemic model with voluntary vaccinations. J. Math. Biol. 53, 253-272 (2006)

2. Shim, E, Feng, Z, Martcheva, M, Castillo-Chavez, C: An age-structured epidemic model for rotavirus with vaccination. J. Math. Biol. 53, 719-749 (2006)

3. Moneim, IA, Greenhalgh, D: Threshold and stability results for an SIRS epidemic model with a general periodic vaccination strategy. J. Biol. Syst. 13, 131-150 (2005)

4. Greenhalgh, D: Hopf bifurcation in epidemic models with a latent period and nonpermanent immunity. Math. Comput. Model. 25, 85-107 (1997)

5. Greenhalgh, D: Some results for an SEIR epidemic model with density dependence in the death rate. Math. Med. Biol. 9. 67-68 (1992)

6. Li, J, Ma, Z: Qualitative analyses of SIS epidemic model with vaccination and varying total population size. Math Comput. Model. 35, 1235-1243 (2002)

7. Li, J, Ma, Z: Global analysis of SIS epidemic models with variable total population size. Math. Comput. Model. 39 , 1231-1242 (2004)

8. Deng, C, Gao, H: Stability of SVIR system with random perturbations. Int. J. Biomath. 5, 1250025 (2012)

9. Tornatore, E, Buccellato, SM, Vetro, P: Stability of a stochastic SIR system. Physica A 354, 111-126 (2005)

10. Liu, Q: Stability of SIRS system with random perturbations. Physica A 388, 3677-3686 (2009)

11. Ji, C, Jiang, D, Shi, N: Multigroup SIR epidemic model with stochastic perturbation. Physica A 390, 1747-1762 (2011)

12. Gray, A, Greenhalgh, D, Hu, L, Mao, X, Pan, J: A stochastic differential equation SIS epidemic model. SIAM J. Appl. Math. 71(3), 876-902 (2011)

13. Dalal, N, Greenhalgh, D, Mao, X: A stochastic model of AIDS and condom use. J. Math. Anal. Appl. 325, 36-53 (2007)

14. Bandyopadhyay, $M$, Chattopadhyay, J: Ratio-dependent predator-prey model: effect of environmental fluctuation and stability. Nonlinearity 18, 913-936 (2005)

15. Beretta, E, Kolmanovskii, $\mathrm{V}$, Shaikhet, L: Stability of epidemic model with time delays influenced by stochastic perturbations. Math. Comput. Simul. 45, 269-277 (1998)

16. Carletti, M: On the stability properties of a stochastic model for phage-bacteria interaction in open marine environment. Math. Biosci. 175, 117-131 (2002)

17. Yu, J, Jiang, D, Shi, N: Global stability of two-group SIR model with random perturbation. J. Math. Anal. Appl. 360, 235-244 (2009)

18. Beddington, JR, May, RM: Harvesting natural populations in a randomly fluctuating environment. Science 197, 463-465 (1977)

19. Imhof, L, Walcher, S: Exclusion and persistence in deterministic and stochastic chemostat models. J. Differ. Equ. 217, 26-53 (2005)

20. Mao, X: Stochastic Differential Equations and Applications. Horwood, Chichester (1997)

21. Chen, L, Chen, J: Nonlinear Biological Dynamical System. Science Press, Beijing (1993)

22. Higham, DJ: An algorithmic introduction to numerical simulation of stochastic differential equations. SIAM Rev. 43 525-546 (2001)

23. Imhof, L, Walcher, S: Exclusion and persistence in deterministic and stochastic chemostat models. J. Differ. Equ. 217 26-53 (2005) 
24. Zhao, Y, Jiang, D: Dynamics of stochastically perturbed SIS epidemic model with vaccination. Abstr. Appl. Anal. 2013, Article ID 517439 (2013)

25. Fu, J, Han, Q, Lin, Y, Jiang, D: Asymptotic behavior of a multigroup SIS epidemic model with stochastic perturbation. Adv. Differ. Equ. 2015, 84 (2015)

26. Golmankhaneh, AK, Porghoveh, NA, Baleanu, D: Mean square solutions of second-order random differential equations by using homotopy analysis method. Rom. Rep. Phys. 65, 350-362 (2013)

27. Jafarian, A, Measoomy Nia, SA, Golmankhaneh, AK, Baleanu, D: Numerical solution of linear integral equations system using the Bernstein collocation method. Adv. Differ. Equ. 2013, 123 (2013)

Submit your manuscript to a SpringerOpen ${ }^{\circ}$ journal and benefit from:

- Convenient online submission

Rigorous peer review

- Immediate publication on acceptance

- Open access: articles freely available online

- High visibility within the field

- Retaining the copyright to your article 